\title{
ESTUDOS DE CARACTERIZAÇÃO TECNOLÓGICA EM AMOSTRAS DE MINÉRIO DE FERRO DE BAIXO TEOR
}

\author{
S. S. CARMO ${ }^{1}$, D. ULIANA ${ }^{1}$, H. KAHN ${ }^{1}$ e M. M. L. TASSINARI ${ }^{1}$ \\ ${ }^{1}$ Depto. de Eng. de Minas e de Petróleo da Escola Politécnica - Universidade de São Paulo \\ sheila@lct.poli.usp.br; daniel@Ict.poli.usp.br; henrique@lct.poli.usp.br; manuela@lct.poli.usp.br
}

Artigo submetido em novembro/2013 e aceito em fevereiro/2014

DOI: http://dx.doi.org/10.15628/holos.2014.1772

\section{RESUMO}

O crescimento da produção e do valor do minério de ferro frente a aumentos de demanda tem levado as mineradoras a buscar a ampliação de suas reservas a partir de recursos minerais de baixo teor. 0 intemperismo atuante sobre eles resulta em composições mineralógicas e aspectos de associação mineral diversa, especialmente em relação aos conteúdos de goethita e argilominerais, implicando na necessidade de estudos detalhados para avaliação da qualidade dos produtos passíveis de serem obtidos, quanto a teores de SiO2, Al2O3, P e perda ao fogo, críticos nas especificações metalúrgicas. Neste contexto, duas amostras com diferentes graus de intemperismo e composição mineralógica foram estudadas, visando avaliar a possibilidade de obtenção de produtos dentro das especificações usuais requeridas. Os estudos compreenderam cominuição em duas condições: separações minerais e avaliação dos aspectos mineralógicos por análise de imagens por feixe de elétrons (MLA). A amostra 1 é composta principalmente por hematita, quartzo e goethita e a amostra 2 por magnetita, quartzo e anfibólios. Os estudos mostram que, para uma recuperação de $95 \%$ do ferro contido nos óxidos de ferro, teoricamente, é possível obter-se produto com teor de $66 \%$ para a amostra 1 e $69 \%$ na amostra 2.

PALAVRAS-CHAVE: minério de ferro, caracterização tecnológica, análise de imagens.

\section{TECHNOLOGICAL CHARACTERIZATION STUDIES OF LOW GRADE IRON ORE SAMPLES}

\begin{abstract}
The growth of production and the value of iron ore have led mining companies to seek expansion of its reserves from low-grade mineral resources, where the weathering process lead to varied mineralogy and mineralogical association, especially regarding the contents of goethite and clay minerals. Detailed characterization studies are fundamental to evaluate the products that can be obtained considering the grades of $\mathrm{SiO}_{2}, \mathrm{Al}_{2} \mathrm{O}_{3}, \mathrm{P}$ and loss on ignition, which are critical metallurgical specifications. In this context, this study is based on the characterization of two samples with
\end{abstract}

different degrees of weathering and mineralogical composition to evaluate the possibility of obtaining granulometric products within usual specifications required. The main results were obtained from SEMbased image analysis (MLA). Sample 1 consists primarily of hematite, quartz and goethite, while sample 2 comprises magnetite, quartz and amphibole. Considering the Fe present in the iron oxides, the research indicates a potential recovery of $95 \%$ with $\mathrm{Fe}$ grades of $66 \%$ for sample 1 and $69 \%$ for sample 2

KEYWORDS: iron ore, technological characterization, automated image analysis. 


\section{INTRODUÇÃO}

Nos últimos anos as empresas produtoras de minério de ferro têm investido em estudos de caracterização tecnológica para avaliação da potencialidade de seus recursos minerais, com o objetivo de ampliação de reservas, atendimento a crescimentos de produção, visto que, em cenários de grande demanda, a elevação do preço das "commodities" pode viabilizar o aproveitamento de recursos mais complexos e de teores mais baixos. Para os minérios de ferro de alto teor, normalmente é suficiente apenas uma adequação granulométrica, mas em materiais de baixo teor, estudos mais acurados são necessários. Os minérios de ferro brasileiros apresentam diferentes graus de intemperismo, responsáveis por assembleias mineralógicas variadas, especialmente em relação aos conteúdos de goethita, argilominerais e outros minerais, responsáveis pela diminuição do teor de ferro e aumento significativo dos de sílica, alumínio e fósforo junto ao concentrado, críticos para as especificações metalúrgicas (ROCHA, 2008; COUTO, 2009; COUTO, COSTA et al., 2010).

Neste contexto, estudaram-se duas amostras de composição mineralógica bastante distinta, tanto em relação ao mineral de minério como em relação aos minerais de ganga presentes, com o objetivo de verificar a forma de ocorrência dos óxidos de ferro e suas associações com a ganga contida, com enfoque nos portadores de sílica, alumínio e fósforo, para avaliação da possibilidade de obtenção de produtos granulométricos dentro das especificações usuais requeridas. As atividades realizadas compreenderam cominuições em duas condições, classificações granulométricas, separações minerais e estudos mineralógicos detalhados por microscopia eletrônica de varredura, utilizando análise de imagens por feixe de elétrons (MLA). Os estudos efetuados ao MEV/MLA possibilitam a obtenção de resultados altamente confiáveis, em função da observação detalhada de grande número de partículas, tendo como principais resultados a composição mineralógica, a partição dos elementos químicos de interesse, características de associação e grau de liberação dos minerais presentes, além da recuperação potencial do elemento de interesse, constituindo uma ferramenta extremamente importante para o setor mineral (FANDRICH, GU et al., 2007). Estudos utilizando a mesma metodologia foram realizados, em minérios de ferro, por ULIANA et. al (2011).

\section{MATERIAIS E MÉTODOS}

Inicialmente, em ambas as amostras, avaliou-se a possibilidade de obtenção de produto grosso constituído por minerais de ganga a ser descartado, com consequente redução da quantidade a ser cominuída posteriormente em malha mais fina (minimização nos custos dessa operação), visando geração de produtos granulométricos dentro das especificações químicas correntes de mercado. A amostra 1 (friável) apresentava pequena parcela de material acima de $6,35 \mathrm{~mm}$, razão pela qual foi cominuída abaixo dessa fração (avaliação da possibilidade de obtenção de sinter feed); mesmo procedimento foi usado na amostra 2 (material fresco representado por testemunhos de sondagem).

Para a amostra 1, após essa avaliação, os estudos prosseguiram com a realização de nova cominuição (abaixo de 0,60 mm), seguida de classificação granulométrica e separações minerais em escala de bancada (líquidos densos e em ímã de mão); já para a amostra 2 efetuou-se 
cominuição abaixo de 0,21 mm, classificação granulométrica e separações magnéticas em ímã de mão. Todos os produtos gerados nesse procedimento foram submetidos a análises químicas por fluorescência de raios $X$; difrações de raios $X$ foram efetuadas em produtos específicos. $O$ estudo das características mineralógicas por MEV/MLA foram realizados em ambas as amostras apenas após a cominuição em malha mais fina.

\section{RESULTADOS}

\subsection{Composição química}

A amostra 1 é mais rica em Fe do que a amostra 2 (44,2\% e 36,2\%, respectivamente), ocorrendo o inverso em relação ao $\mathrm{SiO}_{2}$ (41,2\% contra 32,9\%); teores de $\mathrm{Al}_{2} \mathrm{O}_{3}$ situam-se entre 1,39 e $1,85 \%$ e os de $P$ entre $0,05-0,06 \%$ para as 2 amostras. A amostra 2 tem teor de $3,08 \%$ de $\mathrm{CaO}$ e 1,85\% de $\mathrm{MgO}$, enquanto que na amostra 1 esses são inferiores a 0,10\%. A amostra 2 mostra ganho ao fogo, enquanto que a amostra 1 tem perda ao fogo de 2,54\%. Esses resultados estão expostos na Tabela 1.

Tabela 1 - Composição química das amostras estudadas (teores dosados).

\begin{tabular}{cccccccccccc}
\hline Teores (\%) & $\mathbf{F e}$ & $\mathbf{S i O}_{\mathbf{2}}$ & $\mathbf{A l}_{\mathbf{2}} \mathbf{O}_{\mathbf{3}}$ & $\mathbf{P}$ & $\mathbf{M n}$ & $\mathrm{TiO}_{2}$ & $\mathbf{C a O}$ & $\mathbf{M g O}$ & $\mathbf{N a}_{2} \mathbf{O}$ & $\mathbf{K}_{\mathbf{2}} \mathbf{O}$ & $\mathbf{P F}$ \\
\hline Amostra 1 & 44,2 & 32,9 & 1,85 & 0,05 & 0,10 & $<0,10$ & $<0,10$ & $<0,10$ & $<0,10$ & $<0,10$ & 2,54 \\
Amostra 2 & 36,2 & 41,2 & 1,39 & 0,06 & 0,15 & $<0,10$ & 3,08 & 1,85 & $<0,10$ & 0,19 & $\mathrm{gf}$ \\
\hline
\end{tabular}

3.2 Avaliação da possibilidade de obtenção de produto de ganga leve - cominuição grossa

Os resultados obtidos após a cominuição a $-6,35 \mathrm{~mm}$ estão expostos da Tabela 2Tabela até a Tabela 5.

Para a amostra 1 verificou-se, após a execução de separações minerais (líquidos densos), a possibilidade de obtenção de produto constituído por ganga leve correspondente a cerca de $3,4 \%$ em massa na fração $-6,35+0,60 \mathrm{~mm}$, aliado a uma perda de ferro de $2,4 \%$ do total contido na amostra. Abaixo de $0,60 \mathrm{~mm}$, a proporção em massa desse produto aumenta gradativamente para os finos, enquanto que o conteúdo de ferro tende a diminuir, com a geração de produtos (afundados) com teores de Fe superiores a 62\%.

Para a amostra 2, onde há amplo predomínio de magnetita, as separações magnéticas até $0,21 \mathrm{~mm}$ propiciaram a geração de produto não magnético correspondente a $6 \%$ em massa, com perda de ferro da ordem de $2 \%$ do total contido. Abaixo de $0,21 \mathrm{~mm}$ a proporção em massa do produto não magnético aumenta gradativamente para os finos, com geração de produtos magnéticos já com teores mais elevados de Fe (superiores a 55\%). 
Tabela 2 - Distribuição de teores por fração granulométrica - Amostra 1 (-6,35 mm)

\begin{tabular}{|c|c|c|c|c|c|c|c|c|c|c|c|}
\hline \multirow{2}{*}{$\begin{array}{l}\text { Fração } \\
(\mathrm{mm})\end{array}$} & \multicolumn{2}{|c|}{$\%$ Massa } & \multicolumn{5}{|c|}{ Teores (\%) } & \multicolumn{4}{|c|}{ Distribuição no ensaio (\%) } \\
\hline & retida & acum. & $\mathrm{Fe}$ & $\mathrm{SiO}_{2}$ & $\mathrm{Al}_{2} \mathrm{O}_{3}$ & $\mathbf{P}$ & PF & $\mathrm{Fe}$ & $\mathrm{SiO}_{2}$ & $\mathrm{Al}_{2} \mathrm{O}_{3}$ & $\mathbf{P}$ \\
\hline$-6,35+1,00$ & 41,1 & 41,1 & 58,1 & 12,2 & 0,90 & 0,05 & 2,30 & 52,8 & 16,2 & 20,7 & 30,2 \\
\hline$-1,00+0,60$ & 3,8 & 44,9 & 56,5 & 14,6 & 0,85 & 0,05 & 2,12 & 4,7 & 1,8 & 1,8 & 2,6 \\
\hline$-0,60+0,30$ & 5,1 & 50,0 & 52,4 & 21,3 & 0,64 & 0,04 & 1,49 & 5,9 & 3,5 & 1,8 & 2,8 \\
\hline$-0,30+0,15$ & 12,1 & 62,0 & 40,3 & 40,3 & 0,66 & 0,03 & 1,02 & 10,8 & 15,7 & 4,5 & 4,9 \\
\hline$-0,15$ & 38,0 & 100,0 & 30,8 & 51,3 & 3,35 & 0,11 & 3,33 & 25,8 & 62,8 & 71,2 & 59,4 \\
\hline Total & 100,0 & & 45,2 & 31,0 & 1,79 & 0,07 & 2,49 & 100,0 & 100,0 & 100,0 & 100,0 \\
\hline
\end{tabular}

Tabela 3 - Resultados das separações por líquidos densos após cominuição a -6,35 mm - Amostra 1

\begin{tabular}{|c|c|c|c|c|c|c|c|c|c|c|c|c|c|c|c|}
\hline \multirow[t]{2}{*}{ Produto } & \multicolumn{2}{|c|}{ \% Massa } & \multicolumn{5}{|c|}{ Teores (\%) } & \multicolumn{4}{|c|}{ Distrib. no ensaio (\%) } & \multicolumn{4}{|c|}{ Distrib. na amostra (\%) } \\
\hline & ens. & amos. & $\mathrm{Fe}$ & $\mathrm{SiO}_{2}$ & $\mathrm{Al}_{2} \mathrm{O}_{3}$ & $\mathbf{P}$ & PF & $\mathrm{Fe}$ & $\mathrm{SiO}_{2}$ & $\mathrm{Al}_{2} \mathrm{O}_{3}$ & $\mathbf{P}$ & $\mathrm{Fe}$ & $\mathrm{SiO}_{2}$ & $\mathrm{Al}_{2} \mathrm{O}_{3}$ & $\mathbf{P}$ \\
\hline \multicolumn{16}{|l|}{$-6,35+1,00$} \\
\hline Flut. & 7,2 & 3,0 & 34,4 & 41,7 & 2,12 & 0,08 & 5,39 & 4,3 & 24,5 & 16,9 & 11,2 & 2,2 & 4,0 & 3,5 & 3,4 \\
\hline Afund. & 92,8 & 38,2 & 59,9 & 9,95 & 0,80 & 0,05 & 2,06 & 95,7 & 75,5 & 83,1 & 88,8 & 50,6 & 12,2 & 17,2 & 26,8 \\
\hline Total calcul. & 100,0 & 41,1 & 58,1 & 12,2 & 0,90 & 0,05 & 2,30 & 100,0 & 100,0 & 100,0 & 100,0 & 52,8 & 16,2 & 20,7 & 30,2 \\
\hline \multicolumn{16}{|l|}{$-1,00+0,60$} \\
\hline Flut. & 11,0 & 0,4 & 12,4 & 77,7 & 0,86 & 0,04 & 2,35 & 2,4 & 58,9 & 11,2 & 10,0 & 0,1 & 1,0 & 0,2 & 0,3 \\
\hline Afund. & 89,0 & 3,4 & 62,0 & 6,72 & 0,85 & 0,05 & 2,09 & 97,6 & 41,1 & 88,8 & 90,0 & 4,6 & 0,7 & 1,6 & 2,3 \\
\hline Total calcul. & 100,0 & 3,8 & 56,5 & 14,6 & 0,85 & 0,05 & 2,12 & 100,0 & 100,0 & 100,0 & 100,0 & 4,7 & 1,8 & 1,8 & 2,6 \\
\hline \multicolumn{16}{|l|}{$-0,60+0,30$} \\
\hline Flut. & 17,7 & 0,9 & 3,4 & 94,3 & 0,31 & 0,01 & 0,24 & 1,1 & 78,1 & 8,6 & 4,6 & 0,1 & 2,7 & 0,2 & 0,1 \\
\hline Afund. & 82,3 & 4,2 & 62,9 & 5,68 & 0,71 & 0,04 & 1,76 & 98,9 & 21,9 & 91,4 & 95,4 & 5,8 & 0,8 & 1,7 & 2,7 \\
\hline Total calcul. & 100,0 & 5,1 & 52,4 & 21,3 & 0,64 & 0,04 & 1,49 & 100,0 & 100,0 & 100,0 & 100,0 & 5,9 & 3,5 & 1,8 & 2,8 \\
\hline \multicolumn{16}{|l|}{$-0,30+0,15$} \\
\hline Flut. & 39,9 & 4,8 & 2,09 & 95,9 & 0,16 & 0,01 & 0,12 & 2,1 & 94,9 & 9,8 & 8,7 & 0,2 & 14,9 & 0,4 & 0,4 \\
\hline Afund. & 60,1 & 7,3 & 65,6 & 3,40 & 1,00 & 0,04 & 1,62 & 97,9 & 5,1 & 90,2 & 91,3 & 10,5 & 0,8 & 4,0 & 4,5 \\
\hline Total calcul. & 100,0 & 12,1 & 40,3 & 40,3 & 0,66 & 0,03 & 1,02 & 100,0 & 100,0 & 100,0 & 100,0 & 10,8 & 15,7 & 4,5 & 4,9 \\
\hline
\end{tabular}

Tabela 4 - Distribuição de teores por fração granulométrica - Amostra 2 (-6,35 mm)

\begin{tabular}{|c|c|c|c|c|c|c|c|c|c|c|c|c|c|c|}
\hline \multirow{2}{*}{$\begin{array}{c}\text { Fração } \\
\text { (mm) }\end{array}$} & \multicolumn{2}{|c|}{ \% Massa } & \multicolumn{6}{|c|}{ Teores (\%) } & \multicolumn{6}{|c|}{ Distribuição no ensaio (\%) } \\
\hline & retida & acum. & $\mathrm{Fe}$ & $\mathrm{SiO}_{2}$ & $\mathrm{Al}_{2} \mathrm{O}_{3}$ & $\mathbf{P}$ & $\mathrm{CaO}$ & MgO & $\mathrm{Fe}$ & $\mathrm{SiO}_{2}$ & $\mathrm{Al}_{2} \mathrm{O}_{3}$ & $\mathbf{P}$ & $\mathrm{CaO}$ & MgO \\
\hline$-6,35+1,00$ & 84,7 & 84,7 & 36,4 & 40,6 & 1,30 & 0,06 & 3,04 & 1,95 & 84,5 & 85,2 & 84,5 & 82,4 & 84,5 & 89,6 \\
\hline$-1,00+0,15$ & 6,1 & 90,8 & 35,8 & 41,5 & 0,98 & 0,06 & 3,28 & 1,78 & 6,0 & 6,3 & 4,6 & 6,2 & 6,6 & 5,9 \\
\hline$-0,15$ & 9,2 & 100,0 & 37,6 & 37,1 & 1,53 & 0,07 & 2,95 & 0,89 & 9,5 & 8,5 & 10,9 & 11,4 & 8,9 & 4,5 \\
\hline Total & 100,0 & & 36,5 & 40,4 & 1,30 & 0,06 & 3,05 & 1,84 & 100,0 & 100,0 & 100,0 & 100,0 & 100,0 & 100,0 \\
\hline
\end{tabular}

Tabela 5 - Resultados das separações magnéticas após cominuição a -6,35 mm - Amostra 2

\begin{tabular}{|c|c|c|c|c|c|c|c|c|c|c|c|c|c|c|c|c|c|c|}
\hline \multirow[t]{2}{*}{ Produto } & \multicolumn{2}{|c|}{ \% Massa } & \multicolumn{6}{|c|}{ Teores (\%) } & \multicolumn{5}{|c|}{ Distribuição no ensaio (\%) } & \multicolumn{5}{|c|}{ Distrib. na amostra (\%) } \\
\hline & ens. & amos. & $\mathrm{Fe}$ & $\mathrm{SiO}_{2}$ & $\mathrm{Al}_{2} \mathrm{O}_{3}$ & $\mathbf{P}$ & $\mathrm{CaO}$ & MgO & $\mathrm{Fe}$ & $\mathrm{SiO}_{2}$ & $\mathrm{Al}_{2} \mathrm{O}_{3}$ & $\mathbf{P}$ & $\mathrm{CaO}$ & $\mathrm{Fe}$ & $\mathrm{SiO}_{2}$ & $\mathrm{Al}_{2} \mathrm{O}_{3}$ & $\mathbf{P}$ & $\mathrm{CaO}$ \\
\hline \multicolumn{19}{|l|}{$-6,35+1,00$} \\
\hline Mag & 94,3 & 79,8 & 37,9 & 38,7 & 1,21 & 0,06 & 2,98 & 1,93 & 98,1 & 89,9 & 88,4 & 97,5 & 92,4 & 82,9 & 76,6 & 74,7 & 80,3 & 78,1 \\
\hline Não mag & 5,7 & 4,9 & 12,3 & 71,7 & 2,62 & 0,02 & 4,01 & 2,14 & 1,9 & 10,1 & 11,6 & 2,5 & 7,6 & 1,6 & 8,6 & 9,8 & 2,1 & 6,4 \\
\hline Total calc & 100,0 & 84,7 & 36,4 & 40,6 & 1,30 & 0,06 & 3,04 & 1,95 & 100,0 & 100,0 & 100,0 & 100,0 & 100,0 & 84,5 & 85,2 & 84,5 & 82,4 & 84,5 \\
\hline \multicolumn{19}{|l|}{$-1,00+0,21$} \\
\hline Mag & 78,8 & 4,0 & 41,4 & 34,3 & 0,95 & 0,06 & 2,93 & 1,88 & 94,4 & 62,8 & 73,3 & 86,6 & 68,6 & 4,5 & 3,4 & 2,9 & 4,5 & 3,8 \\
\hline Não mag & 21,2 & 1,1 & 9,2 & 75,4 & 1,28 & 0,04 & 4,99 & 1,92 & 5,6 & 37,2 & 26,7 & 13,4 & 31,4 & 0,3 & 2,0 & 1,1 & 0,7 & 1,8 \\
\hline Total calc & 100,0 & 5,1 & 34,5 & 43,0 & 1,02 & 0,06 & 3,37 & 1,89 & 100,0 & 100,0 & 100,0 & 100,0 & 100,0 & 4,8 & 5,4 & 4,0 & 5,2 & 5,6 \\
\hline \multicolumn{19}{|l|}{$-0,21+0,15$} \\
\hline Mag & 69,9 & 0,7 & 55,5 & 17,7 & 0,64 & 0,05 & 1,68 & 0,99 & 93,4 & 35,8 & 56,8 & 71,5 & 41,0 & 1,1 & 0,3 & 0,4 & 0,7 & 0,4 \\
\hline Não mag & 30,1 & 0,3 & 9,1 & 73,6 & 1,13 & 0,05 & 5,61 & 1,85 & 6,6 & 64,2 & 43,2 & 28,5 & 59,0 & 0,1 & 0,6 & 0,3 & 0,3 & 0,6 \\
\hline Total calc & 100,0 & 1,1 & 41,5 & 34,5 & 0,79 & 0,05 & 2,86 & 1,25 & 100,0 & 100,0 & 100,0 & 100,0 & 100,0 & 1,2 & 0,9 & 0,6 & 1,0 & 1,0 \\
\hline
\end{tabular}


3.3 Avaliação da possibilidade de obtenção de produtos após cominuição em malha mais fina

As características dos produtos passíveis de serem obtidos para a amostra 1, após cominuição abaixo de 0,60 mm, estão expostas na Tabela 6 e para a amostra 2, cominuída abaixo de 0,21 mm, na Tabela 7. Na amostra 1 o produto correspondente ao sinter feed (fração 0,60+0,15 mm) representa cerca de $79 \%$ em massa no ensaio (33,3\% em relação à amostra) e tem teor médio de $\mathrm{Fe}$ de $64,9 \%$ (94,4\% do total do ensaio; $48,5 \%$ em relação à amostra), mantendo teores residuais de $3,73 \%$ de $\mathrm{SiO}_{2}, 0,99 \%$ de $\mathrm{Al}_{2} \mathrm{O}_{3}, 0,09 \%$ de $\mathrm{P}$ e $1,20 \%$ de PF.

Tabela 6 - Características dos produtos passíveis de obtenção após cominuição a -0,60 mm - Amostra 1

\begin{tabular}{|c|c|c|c|c|c|c|c|c|c|c|c|c|}
\hline \multirow{2}{*}{$\begin{array}{c}\text { Fração } \\
\text { (mm) }\end{array}$} & \multirow{2}{*}{ Produto } & \multicolumn{2}{|c|}{ \% Massa } & \multicolumn{5}{|c|}{ Teores (\%) } & \multicolumn{4}{|c|}{ Distribuição (\%) } \\
\hline & & ens. & amos. & $\mathrm{Fe}$ & $\mathrm{SiO}_{2}$ & $\mathrm{Al}_{2} \mathrm{O}_{3}$ & $\mathbf{P}$ & PF & $\mathrm{Fe}$ & $\mathrm{SiO}_{2}$ & $\mathrm{Al}_{2} \mathrm{O}_{3}$ & $\mathbf{P}$ \\
\hline \multirow[t]{4}{*}{$-0,60+0,15$} & Alimentação & 100,0 & 42,2 & 54,2 & 18,7 & 1,06 & 0,08 & 1,55 & 51,3 & 25,6 & 20,7 & 44,7 \\
\hline & AF Mag imã mão & 78,9 & 33,3 & 64,9 & 3,73 & 0,99 & 0,09 & 1,20 & 48,5 & 4,0 & 15,2 & 38,1 \\
\hline & AF N-Mag imã mão & 4,9 & 2,1 & 51,2 & 10,3 & 3,15 & 0,19 & 10,2 & 2,4 & 0,7 & 3,1 & 5,4 \\
\hline & Flutuado & 16,2 & 6,9 & 3,27 & 93,9 & 0,76 & 0,01 & 0,65 & 0,5 & 20,8 & 2,4 & 1,3 \\
\hline \multirow[t]{4}{*}{$-0,15+0,037$} & Alimentação & 100,0 & 40,6 & 35,0 & 47,6 & 0,86 & 0,03 & 1,21 & 31,9 & 61,5 & 16,0 & 30,3 \\
\hline & AF Mag imã mão & 47,1 & 19,2 & 66,6 & 2,25 & 1,06 & 0,05 & 1,28 & 28,6 & 1,4 & 9,3 & 19,7 \\
\hline & AF N-Mag imã mão & 6,1 & 2,5 & 42,8 & 25,4 & 3,30 & 0,14 & 8,44 & 2,4 & 2,0 & 3,7 & 7,7 \\
\hline & Flutuado & 46,8 & 19,0 & 2,16 & 96,3 & 0,35 & 0,01 & 0,20 & 0,9 & 58,1 & 3,0 & 2,9 \\
\hline \multirow[t]{4}{*}{$-0,037+0,020$} & Alimentação & 100,0 & 7,1 & 43,3 & 32,2 & 2,26 & 0,06 & 3,52 & 6,8 & 7,7 & 8,3 & 6,4 \\
\hline & AF Mag imã mão & 56,8 & 4,0 & 63,5 & 4,15 & 1,90 & 0,07 & 3,08 & 5,6 & 0,6 & 4,0 & 3,8 \\
\hline & AF N-Mag imã mão & 13,8 & 1,0 & 46,6 & 17,6 & 4,50 & 0,15 & 9,43 & 1,0 & 0,6 & 2,3 & 2,1 \\
\hline & Flutuado & 29,4 & 2,1 & 2,56 & 93,5 & 1,92 & 0,01 & 1,59 & 0,1 & 6,5 & 2,1 & 0,4 \\
\hline
\end{tabular}

Tabela 7 - Características dos produtos passíveis de obtenção após cominuição a -0,21 mm - Amostra 2

\begin{tabular}{|c|c|c|c|c|c|c|c|c|c|c|c|c|c|c|c|c|}
\hline \multirow{2}{*}{$\begin{array}{c}\text { Fração } \\
\text { (mm) }\end{array}$} & \multirow{2}{*}{ Produto } & \multicolumn{2}{|c|}{ \% Massa } & \multicolumn{7}{|c|}{ Teores (\%) } & \multicolumn{6}{|c|}{ Distribuição (\%) } \\
\hline & & ens. & amos. & $\mathrm{Fe}$ & $\mathrm{SiO}_{2}$ & $\mathrm{Al}_{2} \mathrm{O}_{3}$ & $\mathbf{P}$ & $\mathrm{CaO}$ & MgO & PF & $\mathrm{Fe}$ & $\mathrm{SiO}_{2}$ & $\mathrm{Al}_{2} \mathrm{O}_{3}$ & $\mathbf{P}$ & $\mathrm{CaO}$ & MgO \\
\hline \multirow[t]{3}{*}{$-0,21+0,044$} & Alimentação & 100,0 & 69,5 & 36,9 & 41,5 & 1,09 & 0,04 & 2,56 & 1,45 & $\cdots$ & 69,9 & 71,1 & 57,0 & 46,2 & 58,7 & 55,4 \\
\hline & Mag imã mão & 57,4 & 39,9 & 56,4 & 17,3 & 0,70 & 0,04 & 1,26 & 0,79 & 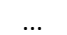 & 61,4 & 17,0 & 21,0 & 29,0 & 16,5 & 17,2 \\
\hline & N-Mag imã mão & 42,6 & 29,6 & 10,5 & 74,2 & 1,61 & 0,03 & 4,31 & 2,34 & 1,35 & 8,5 & 54,2 & 36,1 & 17,2 & 42,1 & 38,2 \\
\hline \multirow[t]{3}{*}{$-0,044+0,037$} & Alimentação & 100,0 & 7,7 & 37,5 & 38,3 & 1,44 & 0,04 & 3,28 & 2,13 & $\ldots$ & 7,8 & 7,4 & 8,7 & 5,3 & 8,4 & 9,0 \\
\hline & Mag imã mão & 46,0 & 3,6 & 64,6 & 7,98 & 0,49 & 0,03 & 0,68 & 0,41 & gf & 6,2 & 0,7 & 1,4 & 1,8 & 0,8 & 0,8 \\
\hline & N-Mag imã mão & 54,0 & 4,2 & 14,4 & 64,1 & 2,25 & 0,05 & 5,49 & 3,59 & 1,74 & 1,6 & 6,7 & 7,3 & 3,5 & 7,6 & 8,2 \\
\hline \multirow[t]{3}{*}{$-0,037$} & Alimentação & 100,0 & 22,8 & 35,4 & 38,4 & 1,91 & 0,12 & 4,34 & 2,81 & $\ldots$ & 22,2 & 21,5 & 34,3 & 48,5 & 32,9 & 35,6 \\
\hline & Mag imã mão & 37,0 & 8,4 & 67,3 & 4,49 & 0,41 & 0,03 & 0,52 & 0,31 & gf & 15,6 & 0,9 & 2,7 & 5,0 & 1,5 & 1,5 \\
\hline & N-Mag imã mão & 63,0 & 14,4 & 16,6 & 58,2 & 2,79 & 0,17 & 6,59 & 4,28 & 1,86 & 6,6 & 20,6 & 31,6 & 43,4 & 31,5 & 34,1 \\
\hline
\end{tabular}

O produto relativo ao "pellet feed" (fração $-0,15+0,037 \mathrm{~mm}$ ) representa $47,1 \%$ em massa no ensaio (19,2\% em relação à amostra) e tem teor médio de $66,6 \%$ de $\mathrm{Fe}(89,7 \%$ do total contido no ensaio; $28,6 \%$ do total da amostra), além de $2,25 \%$ de $\mathrm{SiO}_{2}, 1,06 \%$ de $\mathrm{Al}_{2} \mathrm{O}_{3}, 0,05 \%$ de $P$ e $1,28 \%$ de PF.

Para a amostra 2 o produto magnético da fração $-0,21+0,044 \mathrm{~mm}$ representa $57,4 \%$ em massa no ensaio (39,9\% em relação à amostra) e tem teor médio de $56,4 \%$ de Fe $(61,4 \%$ do total da amostra), com teor residual de $\mathrm{SiO}_{2}$ muito elevado, da ordem de $17 \%$, além de $0,70 \%$ de $\mathrm{Al}_{2} \mathrm{O}_{3}$, $0,04 \%$ de $\mathrm{P}, 1,26 \%$ de $\mathrm{CaO}$ e $0,79 \%$ de $\mathrm{MgO}$. Teores de ferro superiores a $64 \%$ só são atingidos nas frações inferiores a $0,044 \mathrm{~mm}$, mantendo teores de $\mathrm{SiO}_{2}$ de até $8 \%$, decrescentes para os finos. 


\subsection{Composição mineralógica obtida a partir dos estudos de MEV/MLA}

A amostra 1 (total $-0,60+0,020 \mathrm{~mm}$ ) é constituída principalmente por óxidos de ferro (55\%; principalmente hematita, e magnetita), quartzo ( $30 \%$ em massa) e goethita ( $13 \%$ em massa), além de caulinita (0,9\% em massa) e traços de outros (0,2\%; albita, anfibólios, ilmenita, óxidos-Mn, calcita, apatita e gibbsita), conforme Tabela 8.

Tabela $O$ conteúdo de óxidos de ferro acima de 0,15 mm varia de 60 a $70 \%$ e entre 41 e 49\% abaixo dessa fração; já os de goethita situam-se entre 12 e 17\% acima de 0,15 mm, entre 8 e $15 \%$ na fração $-0,15+0,044 \mathrm{~mm}$, atingindo $23 \%$ no passante. Os óxidos de ferro são bem cristalizados, com superfície lisa, mostrando-se, por vezes, com fraturas preenchidas por goethita e com diminutas inclusões de $\mathrm{SiO}_{2}$. A goethita ocorre em partículas com superfície maciça ou porosa, mista com quartzo. Apresenta conteúdos variáveis de fósforo, com teor médio da ordem de $0,30 \%$.

A amostra 2 (total $-0,21+0,020 \mathrm{~mm}$ ) é composta por magnetita (43\% em massa), quartzo (29\%) e anfibólios (10\% de grunerita, 7,4\% de actinolita, 4,4\% de hornblenda), além de calcita $(2,3 \%)$, mica (1,7\%) e outros (1,2\%; albita, goethita, ilmenita, óxido-Mn, apatita, gibbsita e pirita).

O conteúdo de magnetita situa-se entre 39 e $49 \%$, o de quartzo entre 18 e $35 \%$, o de grunerita de 7,6 a 9,1\% acima de $0,044 \mathrm{~mm}$ e entre 14 e $17 \%$ no passante, o de actinolita entre 5,6 e $11 \%$ e o de hornblenda entre 3,6 e 6,5\%. A magnetita geralmente é bem cristalizada, com superfície lisa e homogênea, mais raramente rugosa ou fraturada. Ocorre na forma liberada e como inclusões de dimensões variadas associadas ao quartzo e anfibólios, ou mista com esses minerais. Como característica marcante apresenta, muitas vezes, diminutas inclusões de $\mathrm{SiO}_{2}$.

Tabela 8 - Composição mineralógica das amostras estudadas (MEV/MLA)

\begin{tabular}{|c|c|c|c|c|c|c|c|c|c|c|}
\hline & \multirow{2}{*}{$\begin{array}{c}\text { Minerais } \\
\text { (\% em massa) }\end{array}$} & \multicolumn{9}{|c|}{ Fração (mm) } \\
\hline & & $\begin{array}{c}\text { Total } \\
+0,020 \\
\end{array}$ & $-0,60+0,30$ & $-0,30+0,21$ & $-0,21+0,15$ & $-0,15+0,10$ & $-0,10+0,074$ & $-0,074+0,044$ & $-0,044+0,037$ & $-0,037+0,020$ \\
\hline \multirow{5}{*}{ 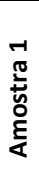 } & óxido de Fe & 55 & 70 & 67 & 60 & 49 & 42 & 48 & 41 & 46 \\
\hline & goethita & 13 & 17 & 15 & 12 & 8,8 & 8,1 & 9,6 & 15 & 23 \\
\hline & quartzo & 30 & 11 & 17 & 28 & 41 & 49 & 42 & 42 & 28 \\
\hline & caulinita & 0,9 & 0,8 & 1,0 & 0,7 & 0,4 & 0,7 & 0,6 & 1,1 & 3,6 \\
\hline & outros* & 0,2 & 0,3 & 0,2 & 0,3 & 0,2 & 0,1 & 0,1 & 0,3 & 0,5 \\
\hline \multirow{8}{*}{ 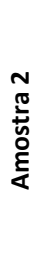 } & magnetita & 43 & & & 39 & 44 & 44 & 49 & 41 & 41 \\
\hline & quartzo & 29 & & & 35 & 34 & 34 & 27 & 23 & 18 \\
\hline & actinolita & 7,4 & & & 8,0 & 6,6 & 5,6 & 5,7 & 10 & 11 \\
\hline & hornblenda & 4,4 & & & 4,9 & 3,6 & 3,6 & 3,6 & 5,0 & 6,5 \\
\hline & grunerita & 10 & & & 9,1 & 8,0 & 7,6 & 8,7 & 14 & 17 \\
\hline & mica & 1,7 & & & 1,3 & 1,4 & 1,3 & 1,7 & 2,2 & 3,1 \\
\hline & calcita & 2,3 & & & 2,0 & 2,1 & 2,4 & 2,6 & 2,7 & 2,6 \\
\hline & outros** & 1,2 & & & 1,3 & 0,8 & 1,3 & 1,4 & 1,3 & 1,1 \\
\hline
\end{tabular}

* albita, anfibólios, ilmenita, óxidos-Mn, calcita, apatita e gibbsita

** albita, goethita, ilmenita, óxido-Mn, apatita, gibbsita

3.5 Partição de Fe, Si, Al e $\mathrm{P}$ nos minerais portadores a partir dos estudos de MEV/MLA

As partições de $\mathrm{Fe}, \mathrm{SiO} 2, \mathrm{Al} 2 \mathrm{O} 3$ e $\mathrm{P}$ nos minerais portadores são mostradas na Figura 1. 

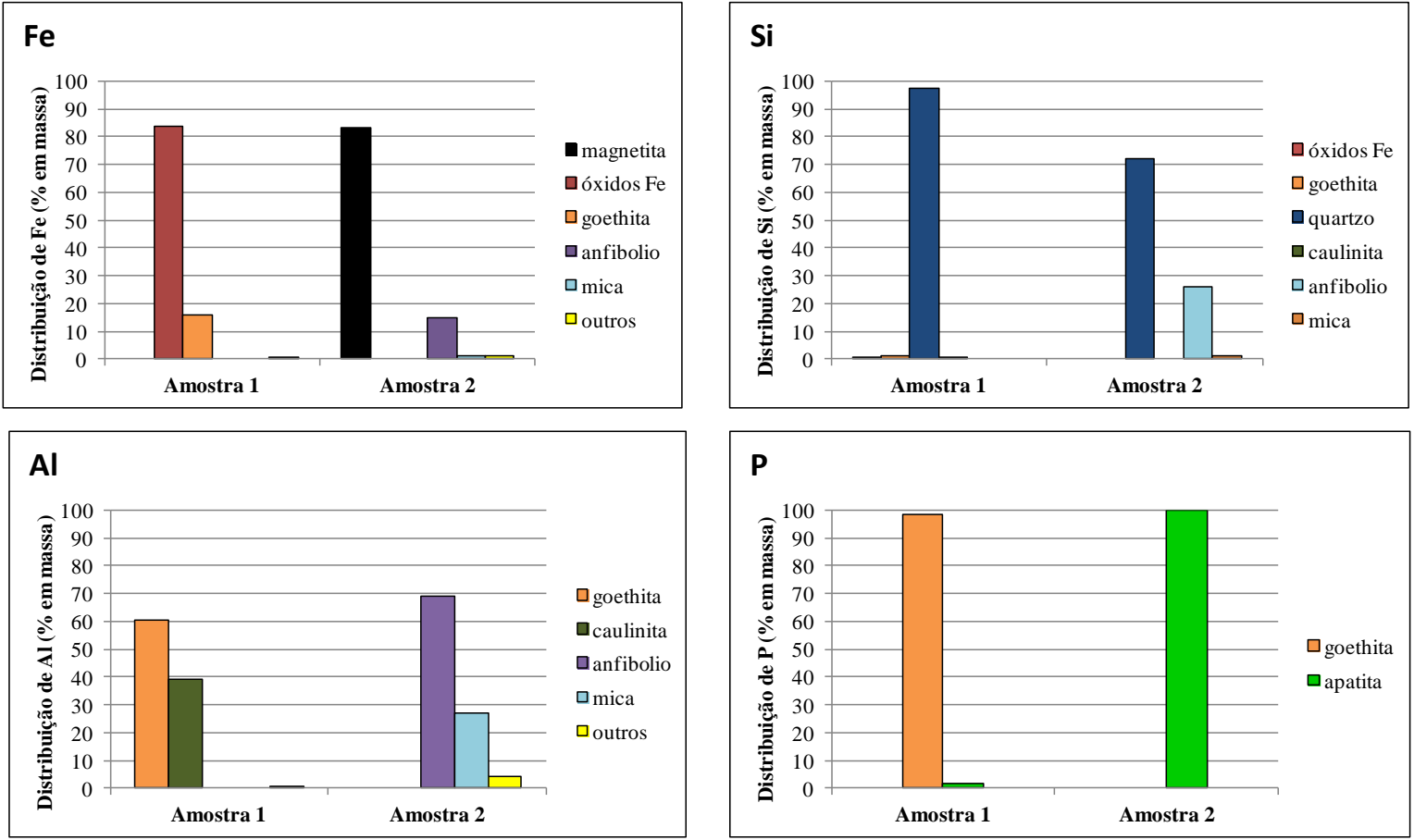

Figura 1 - Distribuição de Fe, Si, Al e P nos minerais portadores (total +0,020 mm)

$\mathrm{Na}$ amostra 1 os óxidos de ferro (hematita e magnetita) respondem por $84 \%$ do Fe total e a goethita por $16 \%$; o quartzo representa $97 \%$ do $\mathrm{SiO}_{2}$ e o restante associa-se aos óxidos de ferro, goethita e caulinita. Goethita e caulinita são as principais fontes de Al e a goethita é também responsável por $99 \%$ do $\mathrm{P}$. Na amostra 2, a magnetita representa $83 \%$ do $\mathrm{Fe}$, os anfibólios $15 \%$ e a mica $1 \%$. O quartzo contém $72 \%$ do total de $\mathrm{SiO}_{2}, 26 \%$ associam-se aos anfibólios e $1 \%$ à mica. A maior parcela do $\mathrm{Al}_{2} \mathrm{O}_{3}$ está nos anfibólios (67\%) e na mica (27\%). Apatita responde pela totalidade do $\mathrm{P}$, a calcita por $47 \%$ do $\mathrm{CaO}$ e os anfibólios pelo restante e ainda por $81 \%$ do $\mathrm{MgO}$.

\subsection{Grau de liberação e associações minerais (MEV/MLA)}

O grau de liberação dos óxidos/hidróxidos de ferro (hematita, magnetita, goethita), considerando como partícula livre aquela com $95 \%$ ou mais do mineral de interesse, está exposto na Figura 2.

Na amostra 1 a liberação (em área) dos óxidos de ferro é de 61\% (total -0,60+0,020 mm); liberações superiores a $90 \%$ só são alcançadas abaixo de $0,037 \mathrm{~mm}$. Na forma mista, esses minerais associam-se à goethita ( $27 \%$ do total de associações binárias) e ao quartzo (7\%). A liberação dos óxidos de ferro em perímetro exposto é inferior à liberação em área, indicando a presença de outros minerais, notadamente goethita, nas bordas das partículas. Já a liberação global dos óxi-hidróxidos de ferro é de $89 \%$, sendo superior a $90 \%$ abaixo de 0,21 $\mathrm{mm}$ e a do quartzo é de $90 \%$. 

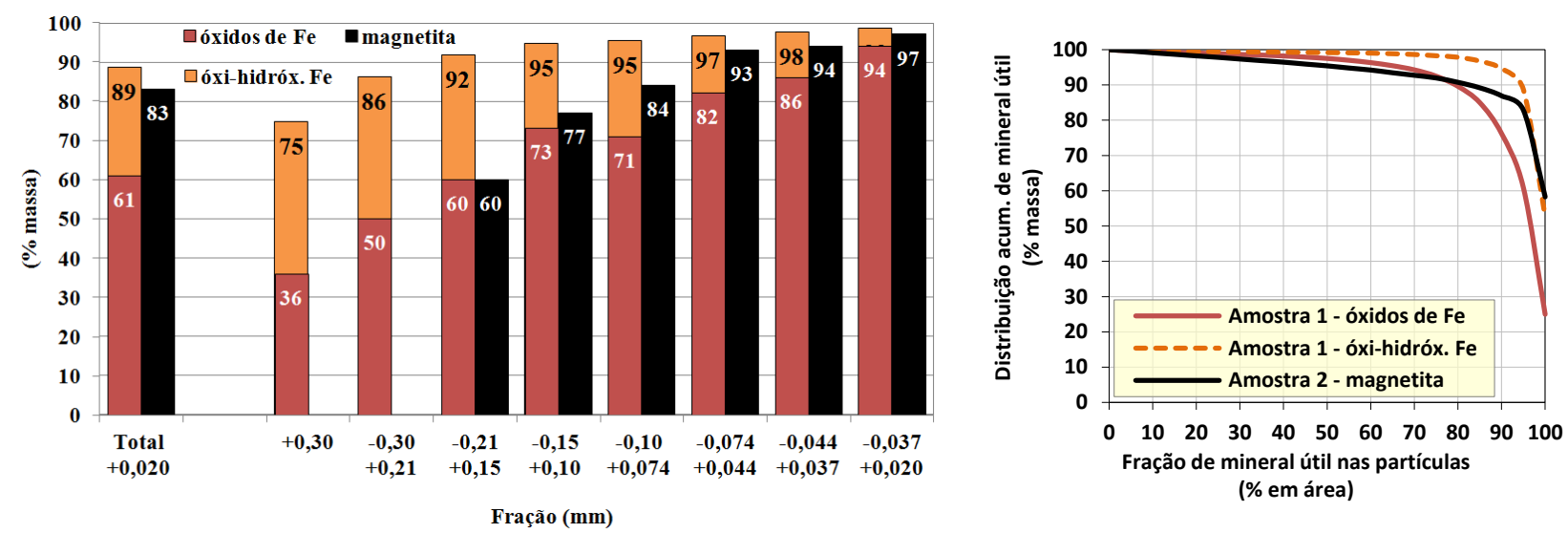

Figura 2 - Grau de liberação dos óxidos / óxi-hidróxidos de Fe (Amostra 1) e magnetita (Amostra 2)

Na amostra 2 a liberação da magnetita (em área) é de $83 \%$, com valores superiores a $90 \%$ apenas abaixo de $0,074 \mathrm{~mm}$. Na forma mista, associa-se ao quartzo em partículas binárias (6,2\% do total), anfibólios (4\% em massa) e à mica (1,6\%). A liberação dos óxidos de ferro em perímetro exposto é inferior à liberação em área, indicando a presença de outros minerais, notadamente quartzo, nas bordas das partículas. Os anfibólios têm liberação de $63 \%$, com valores inferiores a $54 \%$ acima de $0,074 \mathrm{~mm}$ e superiores a $76 \%$ no passante. Na forma mista ocorrem em partículas binárias com magnetita e quartzo ( $14 \%$ em massa do total, cada). A liberação do quartzo é de $67 \%$, com valores superiores a $77 \%$ apenas abaixo de $0,074 \mathrm{~mm}$. Na forma mista ocorre em partículas binárias, associado à magnetita ( $11 \%$ em massa do total) e aos anfibólios ( $13 \%$ em massa).

\subsection{Teor $x$ distribuição de Fe}

As curvas de teor versus recuperação, obtidas nos estudos de MLA para o material acima de 0,020 mm, considerando-se unicamente o Fe presente na forma de óxidos de ferro e, também, a somatória de óxidos e hidróxidos de ferro, é apresentada na Figura 3. Os estudos mostram que, para uma recuperação de $95 \%$ do total de ferro contido na forma de óxidos de ferro (hematita e/ou magnetita), teoricamente, é possível a obtenção de produto com teor de $66 \%$ de Fe para a amostra 1 e $69 \%$ para a amostra 2 . Considerando-se o total de ferro contido nos óxi-hidróxidos de ferro (hematita, magnetita e goethita) da amostra 1, estima-se ser possível, para a mesma recuperação, a obtenção de produto com teor de $67 \%$ de ferro.

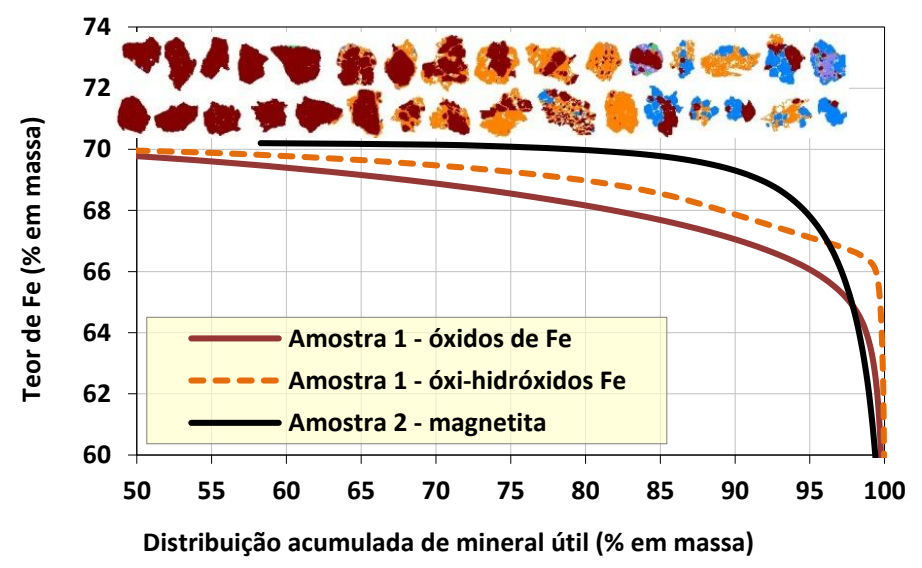

Figura 3 - Curvas de teor versus recuperação dos óxidos e de óxi-hidróxidos de Fe (MLA) 


\section{CONCLUSÕES}

Para a amostra 1 (principalmente hematita) verificou-se a possibilidade de obtenção de produto de ganga leve relativo a 3,5\% em massa para o intervalo $-6,35+0,60 \mathrm{~mm}$, com perda em massa de ferro de $2,5 \%$ do total contido. Para a amostra 2 (principalmente magnetita), é possível até $0,21 \mathrm{~mm}$ a geração de produto não magnético referente a $6 \%$ em massa, carreando $2 \%$ em massa de ferro contido. Os estudos efetuados por MEV/MLA indicam que a amostra 1 (a -0,60 $\mathrm{mm}$ ) é composta por óxidos de ferro ( $55 \%$ de hematita e magnetita), goethita (13\%) e quartzo (30\%). Os óxidos de ferro são responsáveis por $84 \%$ do ferro contido e o restante associa-se à goethita, responsável pelo fósforo da amostra. Os óxidos de ferro apresentam liberação global de $61 \%$. É possível a obtenção de produto sinter feed (fração $-0,60+0,15 \mathrm{~mm}$ ) correspondente a $33,3 \%$ em massa da amostra, com teor de Fe de 64,9\% (48,5\% em relação à amostra) e teores residuais de 3,73\% de $\mathrm{SiO}_{2}, 0,99 \%$ de $\mathrm{Al}_{2} \mathrm{O}_{3}, 0,09 \%$ de $\mathrm{P}$ e $1,20 \%$ de $\mathrm{PF}$ e de produto pellet feed ($0,15+0,037 \mathrm{~mm})$ referente a $19,2 \%$ em massa da amostra, com teor de $66,6 \%$ de Fe $(28,6 \%$ do total da amostra), além de $2,25 \%$ de $\mathrm{SiO}_{2}, 1,06 \%$ de $\mathrm{Al}_{2} \mathrm{O}_{3}, 0,05 \%$ de $\mathrm{P}$ e $1,28 \%$ de PF.

A amostra 2 (a -0,21 mm) é composta por magnetita (43\%), quartzo (29\%) e anfibólios (22\%), além de mica, carbonatos e outros. A magnetita responde por $83 \%$ do total de ferro contido e os anfibólios por $15 \%$. Já o fósforo ocorre na forma de apatita. A magnetita apresenta liberação de $83 \%$, os anfibólios $63 \%$ e o quartzo $67 \%$. Para esta amostra, o produto relativo ao pellet feed (fração $-0,15+0,037 \mathrm{~mm}$ ) representa 43,4\% em massa da amostra e tem teor médio de $57,4 \%$ de $\mathrm{Fe}\left(67,7 \%\right.$ do total da amostra), com teor residual de $\mathrm{SiO}_{2}$ muito elevado, da ordem de $16,5 \%$, além de $0,68 \%$ de $\mathrm{Al}_{2} \mathrm{O}_{3}$ e $0,04 \%$ de $\mathrm{P}, 1,21 \%$ de $\mathrm{CaO}$ e $0,76 \%$ de $\mathrm{MgO}$. Teores de ferro superiores a $64 \%$ são alcançados nas frações com granulação inferior a 0,044 mm, mantendo teores de $\mathrm{SiO}_{2}$ entre 3 e $8 \%$, decrescentes para os finos.

\section{REFERÊNCIAS BIBLIOGRÁFICAS}

1. COUTO, M. L. F. Caracterização de alumínio e fósforo em minério de ferro. 2009.

2. COUTO, M. L. F. et al. Formas de ocorrência de alumínio e fósforo em minérios de ferro. Tecnol. Metal. Mater. Miner., São Paulo, p. 206-209, 2010.

3. FANDRICH, R. et al. Modern SEM-based mineral liberation analysis. International Journal of Mineral Processing, v. 84, p. 310-320, 2007.

4. ROCHA, J. M. P. Definição da Tipologia e Caracterização Mineralógica e Microestrutural dos Itabiritos Anfibolíticos das Minas de Alegria da Samarco Mineração SA MG. 2008. Tese (Doutorado) - Escola de Engenharia, UFMG, Belo Horizonte.

5. ULIANA, D. et al. Process mineralogy studies of low grade iron ores used in the production of pellet feed In: 10th International Congress for Applied Mineralogy, 1-5 August 2011, 2011. ICAM, 2011. p. 717-724. 